\title{
Structure of nano-sized $\mathrm{CeO}_{2}$ material: a combined scattering and spectroscopic investigation
}

\author{
Huw R. Marchbank, ${ }^{a}$ Adam H. Clark, ${ }^{a}$ Timothy I. Hyde, ${ }^{b}$ Helen Y. Playford, ${ }^{c}$ Matthew G. Tucker, ${ }^{c, d}$ \\ David Thompsett, ${ }^{b}$ Janet Fisher, ${ }^{\mathrm{b}}$ Karena W. Chapman, ${ }^{e}$ Kevin A. Beyer, ${ }^{e}$ Manuel Monte $f$, Alessandro \\ Longo. ${ }^{\text {,h }}$ and Gopinathan Sankar ${ }^{\text {** }}$ \\ Dedication ((optional))
}

\begin{abstract}
The structure of several nano-sized ceria, $\mathrm{CeO}_{2}$, systems were investigated using neutron and $\mathrm{X}$-ray diffraction and $\mathrm{X}$-ray absorption spectroscopy. Whilst both diffraction and total pair distribution functions (PDFs) revealed that in all the samples the occupancy of both $\mathrm{Ce}^{4+}$ and $\mathrm{O}^{2-}$ are very close to the ideal stoichiometry, the analysis using reverse Monte Carlo technique revealed significant disorder around oxygen atoms in the nano sized ceria samples in comparison to the highly crystalline NIST standard. In addition, the analysis revealed that the main differences observed in the pair correlations from various $\mathrm{X}$-ray and neutron diffraction techniques were attributed to the particle size of the $\mathrm{CeO}_{2}$ prepared by the reported three methods. Furthermore, detailed analysis of the $\mathrm{Ce}_{3}$ and K-edge EXAFS data support this finding; in particular the decrease in higher shell coordination numbers with respect to the NIST standard, are attributed to differences in particle size.
\end{abstract}

\section{Introduction}

Ceria has been used, over many years, for a range of applications. For example, ceria is widely used in emission based catalytic control within the automotive industry, ${ }^{1-5}$ due to its ability to change oxidation state from $\mathrm{Ce}$ (IV) to $\mathrm{Ce}$ (III) and vice versa at moderate temperatures $\left(<600^{\circ} \mathrm{C}\right)^{3}$ allowing it to store and release oxygen from its structure.

$$
\mathrm{CeO}_{2} \rightleftharpoons \mathrm{CeO}_{2-x}+\frac{x}{2} \mathrm{O}_{2}
$$

a) H. R. Marchbank, A. H. Clark, Prof. Gopinathan Sankar Department of Chemistry, University College London, 20 Gordon Street, London WC1H OAJ (UK)

E-mail: g.sankar@ucl.ac.uk

b) $\mathrm{Dr}$ T. I. Hyde, $\mathrm{Dr}$ D. Thompsett, $\mathrm{Dr}$ J. Fisher Johnson Matthey Technology Centre, Blount's Court, Sonning Common, Reading RG4 9NH (UK)

c) Dr H. Y. Playford, Dr M. G. Tucker

ISIS Facility, Rutherford Appleton Laboratory, Chilton, Didcot, Oxon OX11 0QX (UK)

d) $\operatorname{Dr}$ M. G. Tucker

Diamond Light Source, Harwell Science and Innovation campus, Didcot, Oxon, OX11 ODE (UK) ; current address: Spallation Neutron Source, One Bethel Valley Road, MS-6475, Oak Ridge, TN 37831-6475 (USA)

e) Dr K. W. Chapman, Kevin A. Bayer

X-ray Science Division, Advanced Photon Source, Argonne National Laboratory, (USA)

f) Dr M. Monte

ESRF-The European Synchrotron, CS40220, 38043 Grenoble, Cedex 9 (France) g) $\operatorname{Dr}$ A. Longo

Netherlands Organization for Scientific Research (NWO), ESRF-

h) Thg. Eurngean Synchrotron, CS40220, 38043 Grenoble, Cedex 9

ENRn-4SiMn, Consiglio Nazionale delle Ricerche, Istituto per lo Studio dei Materiali Nanostrutturati, Via Ugo La Malfa 153, 90146 Palermo (Italy)

The fluorite structure (space group $(F m \overline{3} m)$, Ce: 4a, 0, 0, 0 and O: $8 \mathrm{c}, 1 / 4,1 / 4,1 / 4$, Figure 1 ) of ceria allows oxide ions to diffuse easily ${ }^{3}$; ensuring that when either being reduced or oxidised, oxygen may leave or enter the structure. The non-stoichiometry of oxygen within the material creates vacancies within the structure whilst reducing $\mathrm{Ce}$ (IV) to $\mathrm{Ce}$ (III).

$$
\begin{gathered}
O_{o} \rightleftharpoons \frac{1}{2} O_{2}+V_{o}^{*}+2 e^{\prime} \\
2 C e^{4+}+2 e^{\prime} \rightleftharpoons 2 C e^{3+}
\end{gathered}
$$

Furthermore, $\mathrm{CeO}_{2}$ has been used in different areas, for example in hydrocarbon oxidation ${ }^{6}$ and reforming, ${ }^{7}$ and partial oxidation of methan $e^{8}$ in combination with various transition metals. In addition, ceria is used in catalytic wet oxidation reactions, in conjunction with high temperatures and high oxygen pressure, to remove organic waste residues found in water supplies. ${ }^{9}$

Several methods have been reported for the preparation of $\mathrm{CeO}_{2}{ }^{10}$ This area continues to attract attention to improve the performance of this material; here defect structures produced in these systems are thought to be vital for their applications which may depend on the preparation methods. Whilst many of the materials are in crystalline form (some methods yield lower crystallinity compared to the others), it is difficult to rely on one specific crystallographic technique to determine the nature of any defects. For example, X-ray diffraction is routinely used, however, this technique will provide accurate information about cerium atoms and less accurate about the nature of oxygen in the system. Therefore, neutron diffraction methods are desirable for their ability to observe oxygen atoms within the metal oxide material. It has been reported ${ }^{11}$ that, by employing Rietveld ${ }^{12}$ refinement methods of the neutron diffraction data and Pair-Distribution Function (PDF) analysis of the ceria material reveal that there are interstitial oxygen defects within the fluorite structure and these oxide ions provide the mobility that allows ceria to act as an oxygen store for catalysis. 


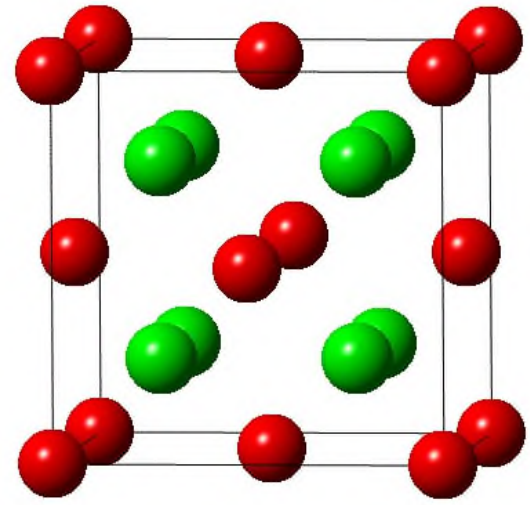

Figure 1: Ceria adopts the fluorite structure (space group Fm-3m, Ce (red): 4a, $0,0,0$ and $O$ (green): $8 \mathrm{c} 1 / 4,1 / 4,1 / 4$ )

Whilst the PDF method provides short, medium and longerrange order information, depending on the range of $r$-space used in the analysis, the data represent a weighted histogram of all interatomic distances in the sample and only through modelling can insight be gained into specific pairwise interactions. ${ }^{13}$ Therefore, an element specific technique, such as X-ray absorption spectroscopy (XAS, which consists of both Xray absorption near edge structure (XANES) and Extended $X$ ray absorption fine structure (EXAFS)) is highly complementary. XANES is widely used to investigate the oxidation state of metal ions present in the samples whereas EXAFS provides information about the coordination environment of the metal ions present in the metal oxide. ${ }^{14}$

In order to fully understand the structure, nature of defects and effects of particle size in ceria prepared by various methods, we used a suite of techniques, in particular XAS (at the Ce $L_{3}$ and K-edges), Rietveld analysis of the X-ray and neutron diffraction data (combined refinement was performed) and PDF analysis of the total scattering data.

\section{Results and Discussion}

First we discuss the results from the combined X-ray and Neutron diffraction data followed by XANES and EXAFS (combined refinement of both $\mathrm{L}_{3}$ and $\mathrm{K}$-edge EXAFS data). Finally we show the analysis of PDF data along with RMC modelling describing the nature of disorder present in the ceria materials.

\section{Joint X-ray and Neutron Rietveld refinement}

Diffraction patterns of all the four samples recorded using Neutron and X-ray radiations are shown in Figure 2. Whilst the diffraction patterns confirm the phase purity of the sample, they show considerable broadening in many of the reflections which indicates that the particle sizes of the synthesised samples are significantly smaller compared to the NIST standard. The combined use of different radiation sources is useful as neutron and $\mathrm{X}$-ray radiation sources interact with the nuclei and electron density, respectively. Therefore, the interaction with many low-Z materials is stronger with neutrons than X-rays. The neutron scattering cross sections for $\mathrm{Ce}$ and $\mathrm{O}$ are 2.94 and 4.232 barns respectively. ${ }^{15}$ Whereas the X-ray scattering cross section for cerium is substantially larger than that of oxygen. ${ }^{16}$ These values show that in X-ray diffraction, the contribution from cerium scattering is a lot greater with respect to oxygen than seen in neutron scattering. This allows the emphasis of both cerium and oxygen depending on the radiation source, giving a more accurate overview of atomic arrangement within ceria.
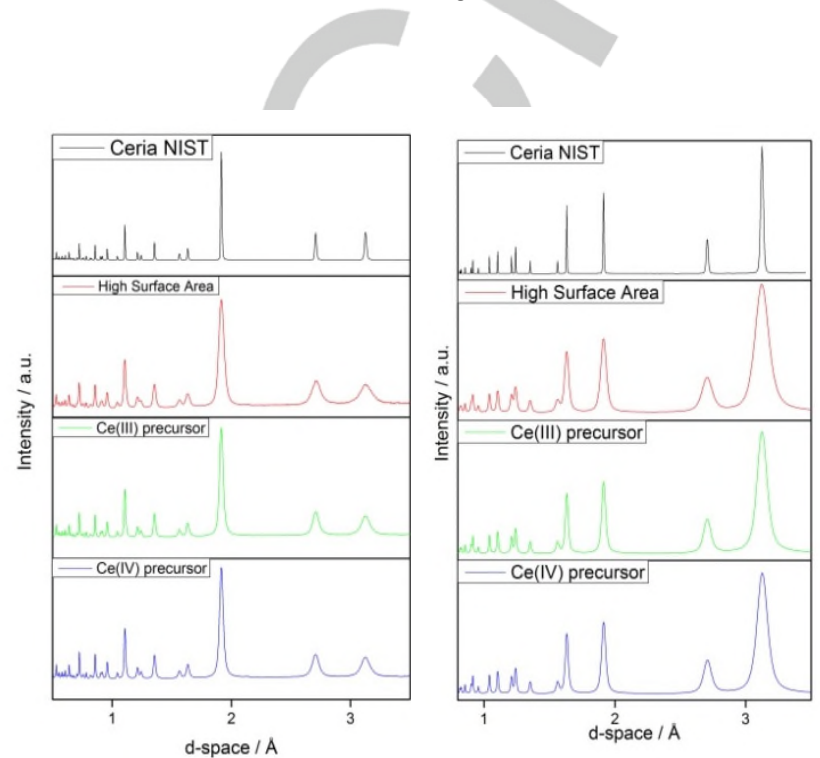

Figure 2: Neutron Diffraction patterns (Left) and X-ray Diffraction patterns (Right) for the 4 samples are shown. These show subtle variations in peak position, intensity and peak broadening indicative of changes in lattice parameter, crystallite size, atomic disorder etc.

Therefore a combined Rietveld refinement of the data, taking both data sets in the analysis, was performed to yield a single structural model for each sample. The Rietveld refinements of all 4 samples show that the Bragg peaks correspond to the ceria fluorite structure (Figure 3). These show that the Bragg peaks only vary subtly in both position and intensity. Error! Reference source not found. shows the refined lattice and structural parameters for all four samples and it appears that they are closely similar. Furthermore, there is peak broadening for the synthesised ceria samples, which is indicative of a smaller crystallite size.

The refinement of the atomic displacement parameters (ADPs) shows that the synthesised samples have greater disorder than the ceria NIST standard. Though it is interesting to note that the ADPs for the Ce (III) precursor ceria sample have similar values for that of the ceria NIST. The refined values of the atom occupancies show that all samples are stoichiometric to within experimental error. 

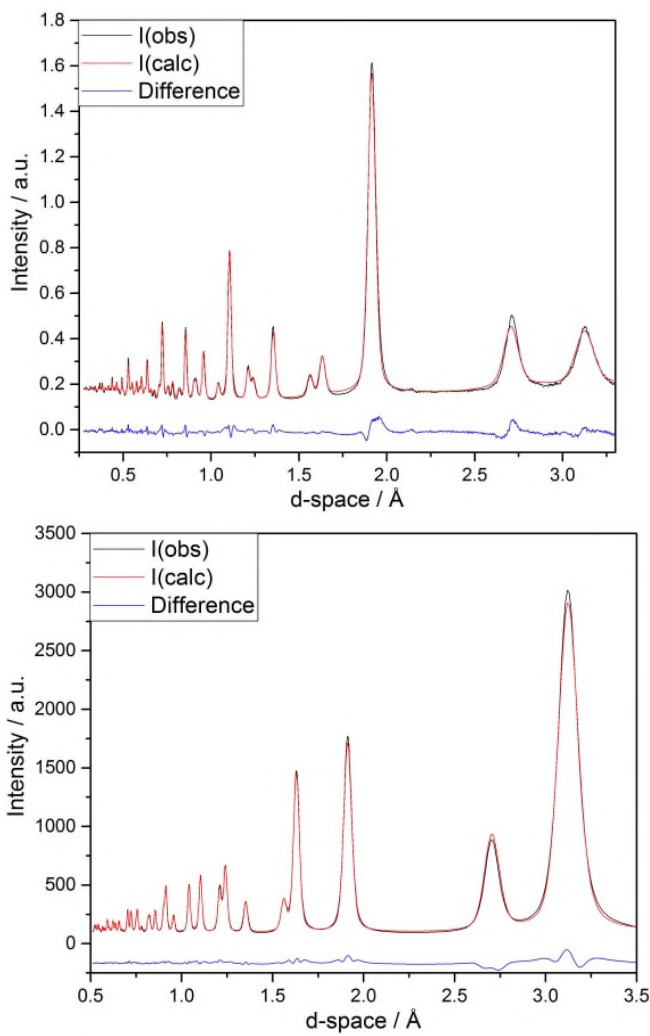

Figure 3: Typical experimental and calculated diffraction patterns obtained from the Rietveld analysis of high surface area ceria material. The upper and lower panels show the neutron and X-ray fits, respectively. Remaining samples are shown in Figures S1-2 of the supporting information

Table 1: Results of Rietveld refinement of joint X-ray and neutron diffraction data of four Ceria materials

\begin{tabular}{lllll}
\hline & Ceria NIST & HSA & $\begin{array}{l}\mathrm{Ce} \\
\text { Precursor }\end{array}$ & $\begin{array}{l}\mathrm{Ce} \\
\text { Precursor }\end{array}$ \\
\hline $\mathrm{a} / \AA$ & $5.4116(51)$ & $5.4099(37)$ & $5.4121(79)$ & $5.4128(31)$ \\
$\mathrm{U}_{\text {iso }} \mathrm{Ce} / \AA^{2}$ & $0.003285(27)$ & $0.00431(4)$ & $0.00364(4)$ & $0.00437(4)$ \\
$\mathrm{U}_{\text {iso }} \mathrm{O} / \AA^{2}$ & $0.00558(18)$ & $0.008199(35)$ & $0.00572(4)$ & $0.007624(26)$ \\
Occupancy & $0.998(1)$ & $0.996(1)$ & $1.00(1)$ & $0.995(1)$ \\
$\mathrm{Ce} /$ a.u. & & & & \\
Occupancy & $1.004(0)$ & $1.005(1)$ & $1.000(1)$ & $1.004(1)$ \\
O / a.u. & & & & 3.88 \\
$\mathrm{R}_{\text {wp }}$ factor & 4.7 & 4.01 & 3.21 & \\
$/ \%$ & & & & \\
\hline
\end{tabular}

\section{XAS analysis}

In order to understand whether any local structural disorder and mixed oxidation states present in the system, we carried out $X$ -

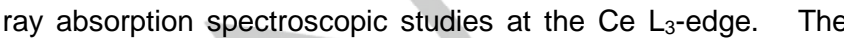
data, shown in Figure 4, reveals that for all ceria samples Ce is found to be predominantly in the $4+$ oxidation state as the characteristic doublet is observed, marked as $C$ and $D$. The XANES region also has a characteristic pre-edge feature, marked feature $\mathrm{A}$ at $5719 \mathrm{eV}$, which has been attributed to delocalisation of the $d$ orbital at the bottom of the conduction band within large clusters, $2 p \rightarrow 5 d$ transition. ${ }^{17}$ Feature $B$ in the XANES is a low-energy shoulder relating to a $2 p \rightarrow 4 f^{1} 5 d_{g} L$ transition at $5724 \mathrm{eV}$ due to the crystal-field splitting of the Ce $5 \mathrm{~d}$ states. ${ }^{18}$ With $C$ being $2 p \rightarrow 4 f^{1} 5 \mathrm{dt}_{2 g} \underline{L}$ transition at $5728 \mathrm{eV}^{18}$. Here the experimental crystal-field energy splitting for the Ce $5 d$ is approximately $4 \mathrm{eV}$ which is in good agreement with previously published experimental and theoretical values of 3.6 $\mathrm{eV}^{19}$ and $4 \mathrm{eV}^{17}$ respectively. $\mathrm{D}$ is then associated to the $2 p \rightarrow 4 f^{0} 5 d$ transition at $5735 \mathrm{eV}$. Figure 4 also shows the XANES observed for Cerium Nitrate, where $\mathrm{Ce}$ is in $3+$ oxidation state, which characteristically shows a singlet at $5724 \mathrm{eV}$ associated to a $2 p \rightarrow 4 f^{1} 5 d$ transition. Ammonium Cerium nitrate is also shown, here the $\mathrm{Ce}$ is in a $\mathrm{Ce}$ (IV) oxidation state, as seen by the distinctive doublet feature, however there is no crystal-field

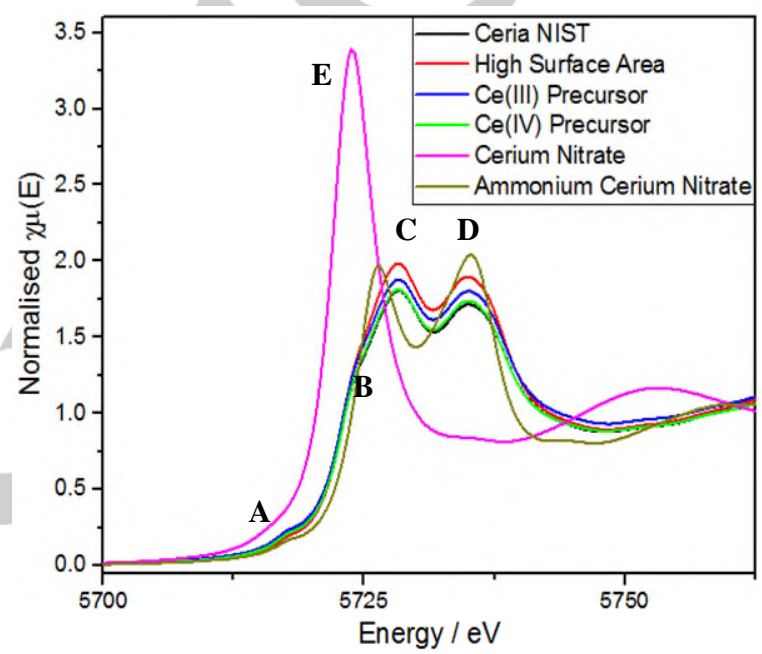

Figure 4: A comparison of the $\mathrm{L}_{3}$-edge for all four Ceria samples and Cerium Nitrate and Ammonium Cerium Nitrate standards with characteristic features labelled. A, pre-edge feature associated to the delocalisation of the $\mathrm{d}$ orbital at the bottom of the conduction band within large clusters. B, a low energy shoulder associated to the crystal-field splitting within the cubic fluorite structure. $\mathbf{C}$ and $\mathbf{D}$ the characteristic doublet for the $2 \mathrm{p} \rightarrow 4 \mathrm{f}^{0,1} 5 \mathrm{~d}$ transitions. $\mathbf{E}$, singlet for the $2 p \rightarrow 4 f^{1} 5 d$ for Ce atom with $3+$ oxidation state $^{19}$

XANES analysis was performed on all samples and the results are given in Error! Reference source not found.. The analysis shows that all the samples have spectral features that appear closely similar in energy for $\mathrm{Ce}^{4+}$ with peak assignments made according the aforementioned transitions using the Athena software package. ${ }^{25} \mathrm{~A}$ large error in position of the pre-edge feature $(A)$ is due the magnitude of this feature being small, as can be seen in Figure 5 below. Using 4 Gaussian peaks is sufficient to replicate the experimental data with excellent agreement in position of the peaks across all samples. The edge-position is given from the position of the arctangent function and can be seen to be consistent over all samples. Given that the peak positions and edge position is consistent over all samples we can conclude that they are all very close to the ideal stoichiometry with only subtle differences. 


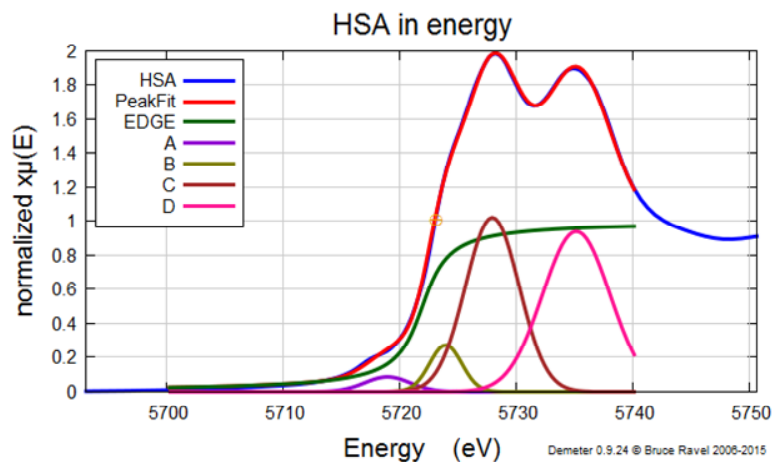

Figure 5: Typical XANES peak fitting showing the HSA Ceria sample with component peaks visible. The edge structure has been fitted with an arctangent function and 4 Gaussian peaks associated to different transitions that have been identified within crystalline Ceria. A, pre-edge feature associated to the delocalisation of the $d$ orbital at the bottom of the conduction band within large clusters. B, a low energy shoulder associated to the crystalfield splitting within the cubic fluorite structure. $C$ and $D$ the characteristic doublet for the $2 p \rightarrow 4 f^{0,1} 5 d$ transitions. Remaining samples shown in Figure S5 of the supporting information.

Table 2: Results of XANES peak fitting using the $\mathrm{Ce} \mathrm{L}_{3}$ edge XAS data

\begin{tabular}{|c|c|c|c|c|}
\hline \multirow{3}{*}{ Peak Position / eV } & \multicolumn{4}{|c|}{ Sample } \\
\hline & Ceria & HSA & $\mathrm{Ce} \quad$ (III) & $\mathrm{Ce}$ \\
\hline & NIST & & Precursor & Precursor \\
\hline \multirow[t]{2}{*}{ Edge Position } & 5722.0 & 5722.4 & 5722.0 & 5722.1 \\
\hline & \pm 0.3 & \pm 0.3 & \pm 0.4 & \pm 0.3 \\
\hline \multirow[t]{2}{*}{ (A) $2 p \rightarrow 5 d$} & 5719 & 5719 & 5719 & 5719 \\
\hline & \pm 3 & \pm 3 & \pm 3 & \pm 3 \\
\hline \multirow[t]{2}{*}{ (B) $2 p \rightarrow 4 f^{1} 5 d e_{g} \underline{L}$} & 5724.1 & 5724.0 & 5724.2 & 5724.0 \\
\hline & \pm 0.2 & \pm 0.5 & \pm 0.2 & \pm 0.3 \\
\hline \multirow[t]{2}{*}{ (C) $2 p \rightarrow 4 f^{1} 5 d t_{2 g} \underline{L}$} & 5728.0 & 5728.0 & $5728.0 \pm$ & 5728.0 \\
\hline & \pm 0.1 & \pm 0.1 & 0.1 & \pm 0.1 \\
\hline \multirow[t]{2}{*}{ (D) $2 p \rightarrow 4 f^{0} 5 d$} & 5735.2 & 5735.2 & $5735.2 \pm$ & 5735.2 \\
\hline & \pm 0.1 & \pm 0.1 & 0.1 & \pm 0.1 \\
\hline
\end{tabular}

\section{Extended X-ray absorption fine structure}

Analysis of the $\mathrm{Ce}_{3}$ edge EXAFS data was performed by assuming that the NIST sample does not suffer from particle size affects and is defect free and as such has been used to determine the amplitude reduction factors. Fitting was undertaken in r-space, however, it was found that a correction is needed to remove the double excitation component in order obtain reliable structural information from the $L_{3}$ edge data.

Therefore, the Correction of the $\chi(k)$ for a double excitation event corresponding to a $2 \mathrm{p}, 4 \mathrm{~d} \rightarrow 5 \mathrm{~d}^{2}$ transition at $5854 \mathrm{eV}^{20}$ was performed by fitting a Lorentzian peak to model the transition resonance to the data with 3 parameters: width, height and position. ${ }^{21}$

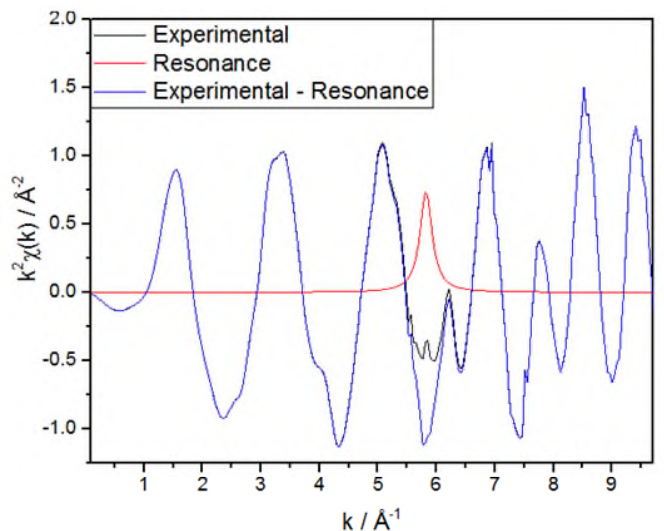

Figure 6: A comparison of initial and final $X(k)$ for the NIST Ceria sample illustrating the Lorentzian peak fitted for removal of the $2 p, 4 d \rightarrow 5 d^{2}$ resonance feature on the $\mathrm{Ce}_{3}$ edge.
Figure 6 illustrates the fitting of the Lorentzian peak showing the initial and final $\chi(k)$. The refinement of the $\mathrm{Ce}-\mathrm{O}$ distance without the resonance correction was found to be 2.30(8) $\AA$ which is in accordance to the 2.30(6) $\AA$ reported by Fonda $\mathrm{el}^{\mathrm{al}^{20}}$ when no resonance correction is made and is significantly small than the crystallographic value of $2.343 \AA$.
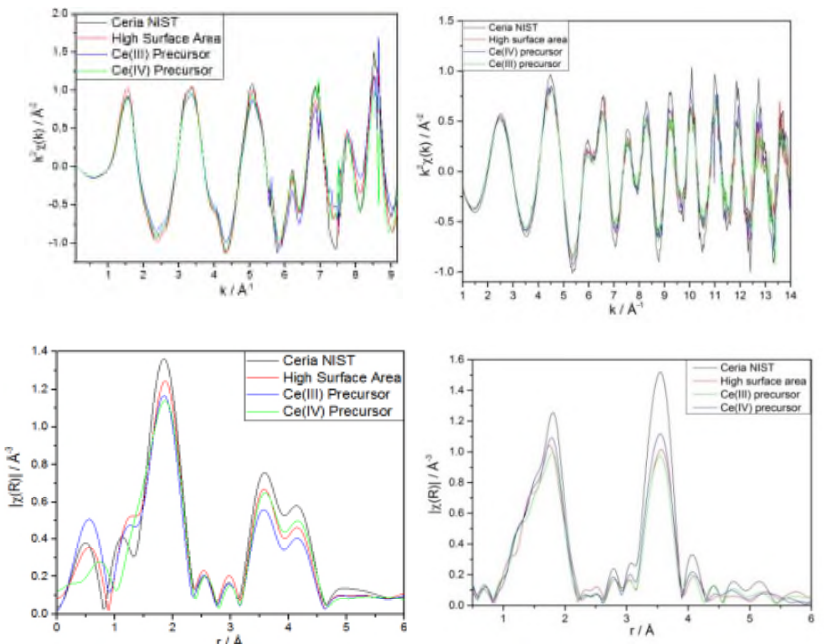
Figure 7: A comparison of the $k^{2} x(k)$ (Top left) and $|x(R)|$ (Bottom left) for al four samples measured at the L3-edge. A comparison of the $k^{2} x(k)$ (Top right) and $|X(R)|$ (Bottom right) for all four samples measured at the K-edge. This shows all have the ceria fluorite structure with subtle subtle variations in intensity and peak position.

The $\mathrm{L}_{3}$-edge spectra provide high quality information regarding the first shell, but cannot provide accurate information regarding second neighbour $\mathrm{Ce}-\mathrm{Ce}$ distance, due to the limited $\mathrm{k}$ range; the $\mathrm{Ce} \mathrm{L}_{2}$ edge overlaps at 6164

$\mathrm{eV}$ or approximately $10.4 \AA^{-1}$ in $\mathrm{k}$. In order to obtain more accurate information on the second and higher neighbour contributions, we measured at the Ce K-edge. However, due to the core-hole lifetime broadening at high energy absorption edge the K-edge data may not provide accurate structural parameters of the first shell.

Therefore, we used the combined refinement of $C e L_{3}$ and $K$ edges to determine accurately the first and higher neighbour correlations. Figure 7 shows a comparison of the $k^{2} \chi(k)$ and $|X(R)|$ of the four samples for the $\mathrm{L}_{3^{-}}$and $\mathrm{K}$-edge respectively. Where the $\chi(k)$ has been Fourier transformed between $\mathrm{k}$ of 2.89 and $9.18 \AA^{-1}$ for the $L_{3}$-edge data and between 2.01 and $14.76 \AA^{-}$ 1 for the K-edge data. The $r$-space data shows that all three samples are closely similar to that of ceria NIST standard, whilst having variations in peak intensity suggesting some alterations in the local structure. A joint edge refinement allows for the emphasis of both first and second shell contributions in r-space. Figure 8 illustrates the experimental data and the calculated fit obtained with this approach. Error! Reference source not found. shows the results obtained from the best fit for all samples.
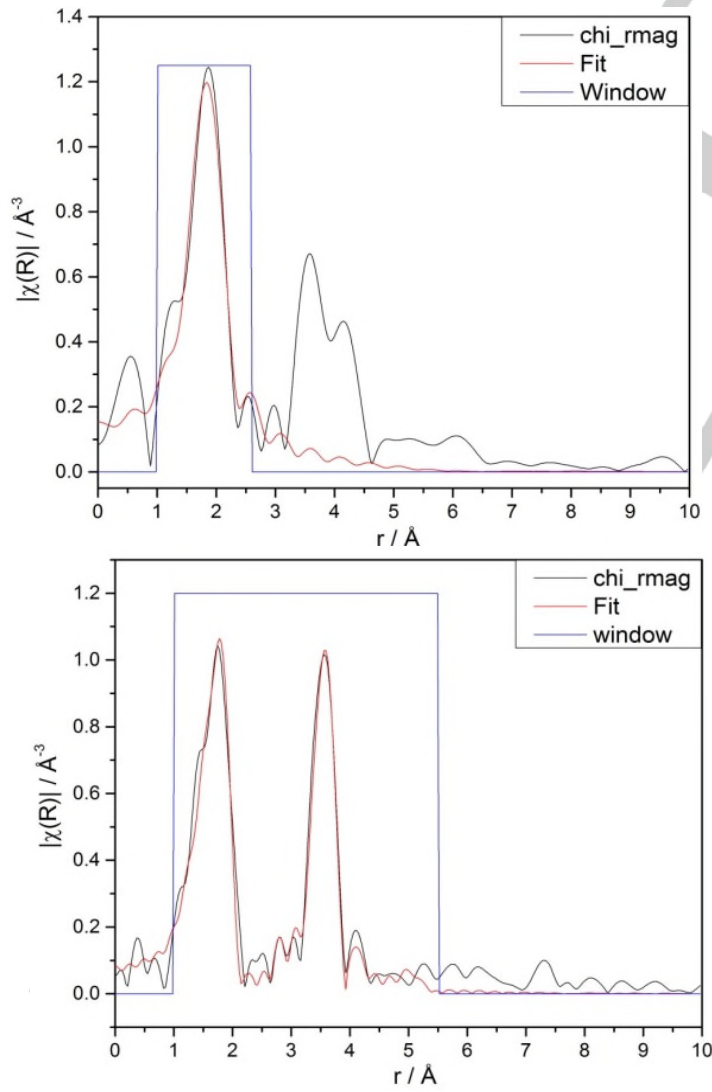

Figure 8: Typical comparison of the EXAFS experimental data and calculated fit for $L_{3}$-edge (Top) and K-edge (Bottom) HSA ceria sample. See Figures S610 of the supporting information for the fits to other samples.

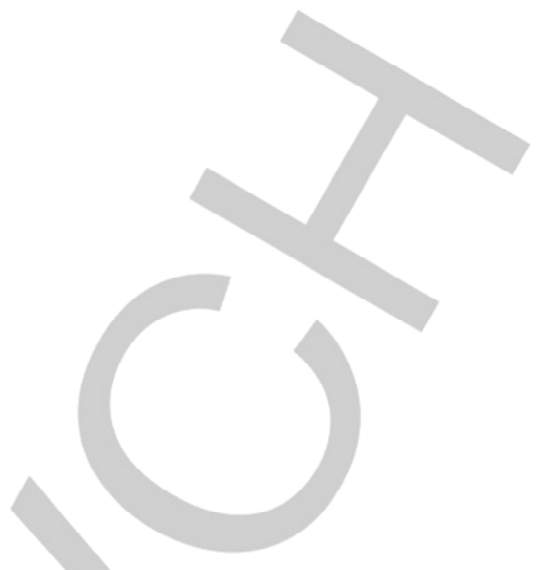

Table 3: Structural parameters obtained using combined EXAFS of the $\mathrm{L}_{3}$ - and K-edges.

\begin{tabular}{lllll}
\hline Sample & Path & $\mathrm{N}$ & $\sigma^{2}$ & $\mathrm{R} / \AA$ \\
\hline Ceria & $\mathrm{O} .1$ & $8^{* *}$ & $0.0044 \pm 0.00023$ & $2.31 \pm 0.004$ \\
NIST & Ce.1 & $12^{* *}$ & $0.00038 \pm 0.00042$ & $3.34 \pm 0.006$ \\
HSA & 0.1 & $8.1 \pm 0.8$ & $0.0064 \pm 0.00028$ & $2.30 \pm 0.003$ \\
& Ce.1 & $9.2 \pm 0.9$ & $0.0045 \pm 0.00065$ & $3.83 \pm 0.008$ \\
Ce (III) & O.1 & $7.8 \pm 0.8$ & $0.0066 \pm 0.00053$ & $2.30 \pm 0.002$ \\
Precursor & Ce.1 & $8.9 \pm 0.9$ & $0.0046 \pm 0.00025$ & $3.84 \pm 0.007$ \\
Ce (IV) & 0.1 & $7.7 \pm 0.8$ & $0.0060 \pm 0.00032$ & $2.31 \pm 0.003$ \\
Precursor & Ce.1 & $9.4 \pm 0.9$ & $0.0042 \pm 0.00064$ & $3.83 \pm 0.007$
\end{tabular}

*refined using K-edge only

**held constant for refinement of amplitude reduction factor

The refinement of the first $\mathrm{Ce}-\mathrm{O}$ path shows that the synthesised samples have larger $\sigma^{2}$ values than the NIST, in agreement with the diffraction results. With respect to crystallographic values, contraction of the first shell is observed with all samples showing smaller than $2.34 \AA$.

For all the synthesised samples the coordination number of the nearest $\mathrm{Ce}-\mathrm{Ce}$ neighbour is significantly lower compared to the NIST standard and $\sigma^{2}$ appears to be slightly higher for the CeCe path compared to ceria NIST. This decrease in coordination number could either be interpreted as due to particle size effect or cation defects present in the system. Since the Ce-O coordination numbers appear to be close that of NIST standard, this implies that the ceria particles are terminated by oxygen.

\section{X-ray and Neutron total scattering data analysis}

In order to evaluate the disorder/defects seen in the XAS results, we carried out pair distribution function (PDF) analysis since it allows the structure to be probed over a larger r-range. PDF analysis is a Rietveld-like approach wherein a structural model is refined to obtain the best possible fit to the PDF, thus producing a model that is biased to local rather than long-range structure. The X-ray and neutron PDF data sets were analysed independently, due to limitations in the analysis software.

Neutron PDF data analysis 
In contrast to the Rietveld refinement, we see different refined parameters when looking at the short and medium range structure. The refinement of the lattice parameter for all samples shows a different trend to that seen in the Rietveld refinement. Error! Reference source not found. shows a comparison of the PDFs for all four samples alongside an example fit undertaken on the Ceria NIST sample. This shows that the local structure of all the samples is consistent with the fluorite structure. In the synthesized samples, the amplitude of the oscillations in $D(r)$ shows significant dampening relative to the NIST sample. Since the instrumental parameters were the same in all cases, this suggests that the HSA ceria, and the samples prepared using $\mathrm{Ce}$ (III) and Ce (IV) precursors have smaller crystallite size.

We also observe that the synthesised samples have higher ADP parameters than that of the reference material. The refined occupancies of both cerium and oxygen atoms are consistent with a fully stoichiometric material.
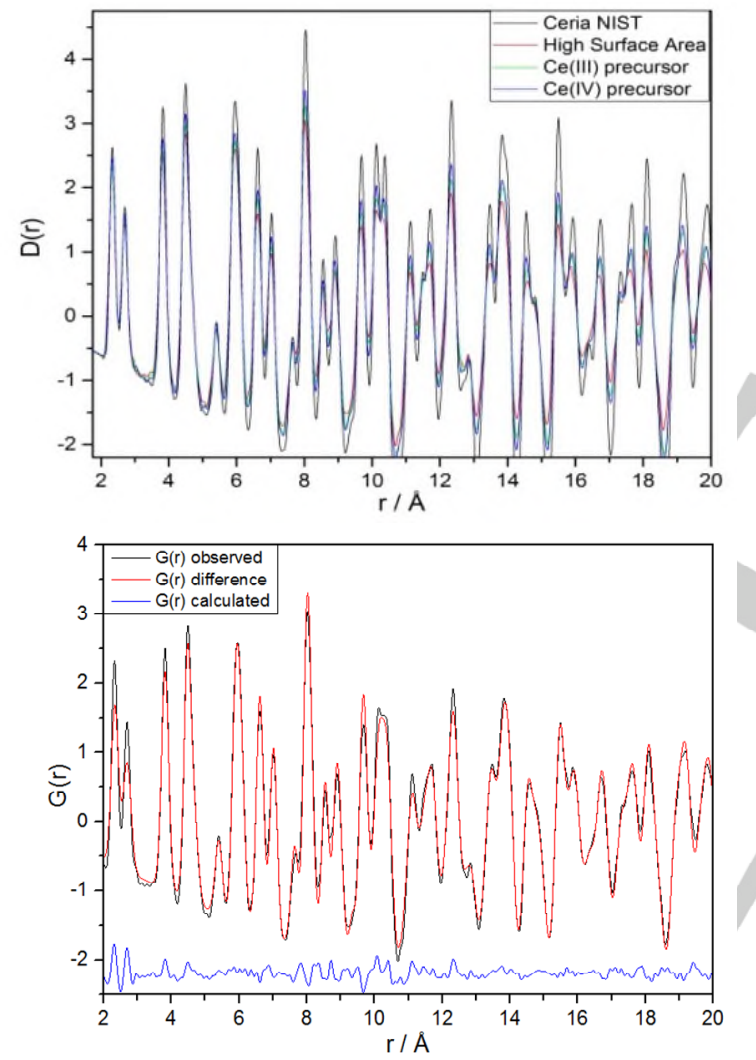

Figure 9: (Top) Comparison of the $G(r)$ of all the four samples obtained from neutron total scattering experiments and in the bottom a typical best fit between experimental and calculated $\mathrm{G}(\mathrm{r})$ for Ceria NIST is shown. Results of the best fit for remaining samples are shown in the supporting information, Figure S3.

\section{X-ray PDF data analysis}

shows a comparison of the PDFs for all four samples alongside an example fit undertaken on the Ceria NIST sample. The modelling of XPDF data was performed with fixed occupancies from the equivalent NPDF analysis. The refined ADPs shows that the HSA, Ce (III) and Ce (IV) precursor ceria samples are more disordered than the reference material. In addition, the dampening of amplitudes in higher $r$ region in $G(r)$ is greater for the synthesised ceria samples compared to ceria NIST corroborating the NPDF results.
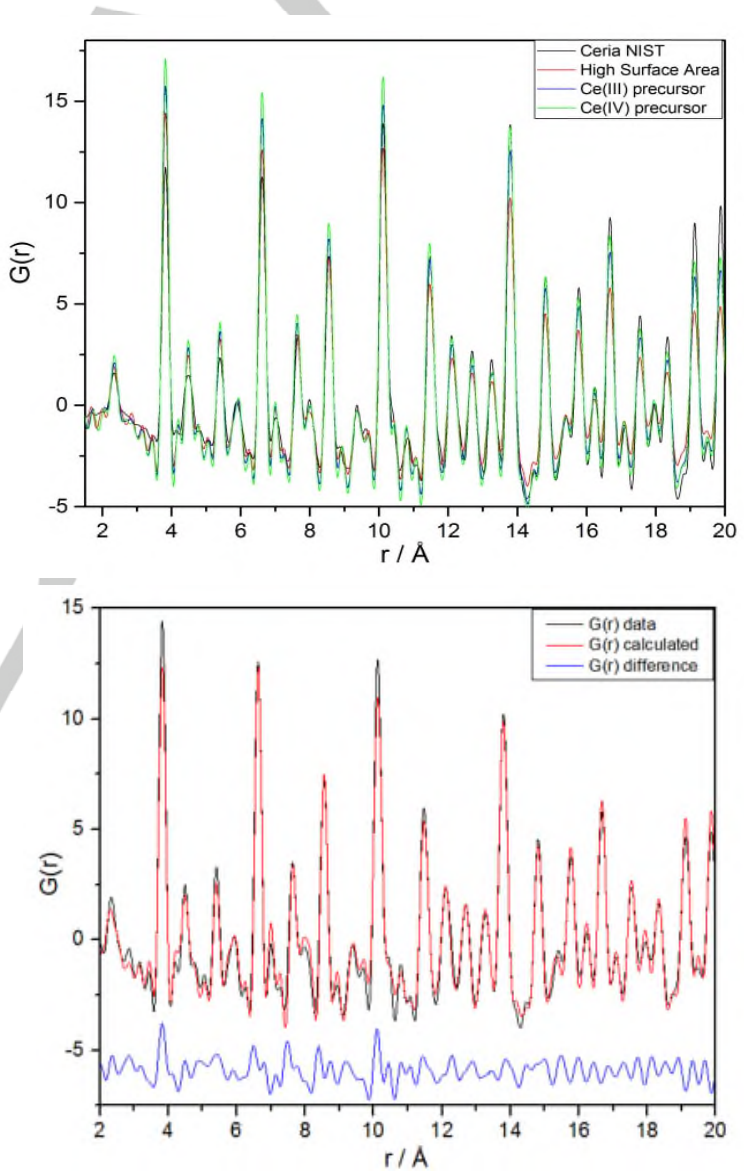

Figure 10: (Top) Comparison of the $G(r)$ of all the four samples obtained from $X$-ray total scattering experiments and in the bottom a typical best fit between experimental and calculated $\mathrm{G}(\mathrm{r})$ for Ceria NIST is shown. Results of the best fit for remaining samples are shown in the supporting information, Figure S4.

\section{Size effect}

Both combined XRD/ND analysis and independent XPDF and NPDF analysis suggest all the system studied here are stoichiometric with site occupancies close to 1 (see Figure 11). However, EXAFS analysis suggests that the synthesised ceria sample have lower coordination number for the Ce-Ce second neighbour pair-correlation. Although the $\mathrm{Ce}-\mathrm{O}$ coordination 
number for samples prepared using $\mathrm{Ce}$ (III) and $\mathrm{Ce}$ (IV) precursors is slightly less than bulk, the obtained values are within error of 4 . When combined, these observations suggest that the particles are oxygen terminated, which would result in full or nearly-full Ce-O shells, but under-coordinated Ce-Ce "shells". The occupancies in Figure 11 based on EXAFS analysis are given in fractions obtained by dividing the obtained coordination number with bulk value ( 8 for $\mathrm{Ce}-\mathrm{O}$ and 12 for $\mathrm{Ce}$ $\mathrm{Ce})$. Therefore, combining the information gained from $\mathrm{X}$-ray and neutron diffraction studies, we propose that the decrease seen in the Ce-Ce coordination number is likely to be due to size effect rather than oxygen defects within the structure.

A comparison of the particle/crystallite size determined from joint ND/XRD, NPDF, XPDF and XAFS analysis shows different values. Sizes obtained from joint $\mathrm{XRD} / \mathrm{ND}$ based analysis is based purely on any crystallites that have the fluorite structure and ignores any contribution from very small and/or amorphous particles. Whereas XPDF, NPDF and EXAFS gives an average information on all types of particle sizes present in the sample, which would explain why the values observed are lower than those seen from joint XRD/ND Rietveld analysis.

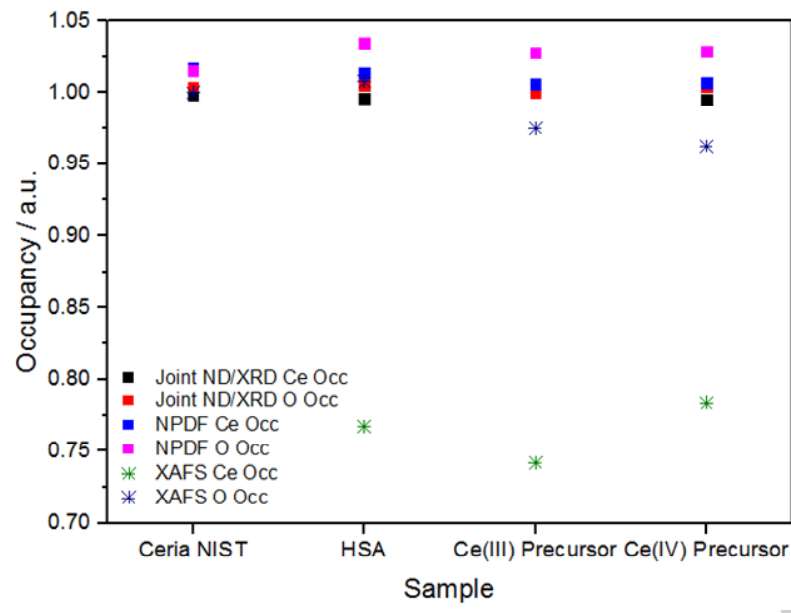

Figure 11: A comparison of the Cerium and Oxygen Occupancies for all four materials analysed with different techniques. In the case of EXAFS, the occupancy number refers to the actual coordination number divided by 8 (for $\mathrm{Ce}-\mathrm{O}$ ) and 12 (for $\mathrm{Ce}-\mathrm{Ce}$ )

The comparison of particle sizes (See Figure 12) obtained from all the different techniques show that HSA, Ce (III) and Ce (IV) precursor ceria samples have a smaller particle size than that of the reference material $\left(\mathrm{CeO}_{2} \mathrm{NIST}\right.$, not shown here as the crystallite size is very large and it is over $10000 \AA$ ).

The results obtained from diffraction based analysis give an average crystallite size assuming spherical particle. Particle sizes obtained from $\mathrm{N}$ - and XPDF are obtained from analysing the dampening in the intensity of $G(r)$ with increasing $r$, assuming a spherical particle. EXAFS analysis assumes Ceria NIST to be a bulk crystalline material and the sizes for the synthesised materials were obtained by considering the change in coordination number of the $\mathrm{Ce}-\mathrm{Ce}$ (second neighbour) with respect to the NIST standard then comparing the refined values with respect to model particles. The differences seen between these methods is that diffraction analysis takes only the crystalline components of the sample and PDF analysis takes into account both crystalline and poorly-crystalline part of the sample. Both methods estimate particle size based on the experimental data; furthermore. EXAFS derived particle size are based on an assumed model to match the coordination number. Furthermore, it has been noted that when cross-correlating EXAFS with other techniques, in particular TEM and XRD EXAFS gives smaller sizes. ${ }^{22}$

\section{Disorder}

To further understand the nature of disorder present in the synthesised samples, we performed reverse Monte Carlo (RMC) analysis of the neutron data using RMCProfile software, since our Rietveld studies clearly suggested that the neutron scattering data are better suited for this analysis due to the X-ray data being dominated by $\mathrm{Ce}-\mathrm{Ce}$ correlations. RMCProfile modelling was performed using a $6 \times 6 \times 6$ supercell constructed from the Rietveld-obtained model. The supercell contained 864 cerium cations and 1728 oxygen anions. For each sample 10 simulations were carried out starting from an initial perfect fluorite structure then the atoms were randomly moved by a set amount to imitate disorder before modelling. The results were averaged over all 10 simulations per sample to minimize the potential for uniqueness problems.

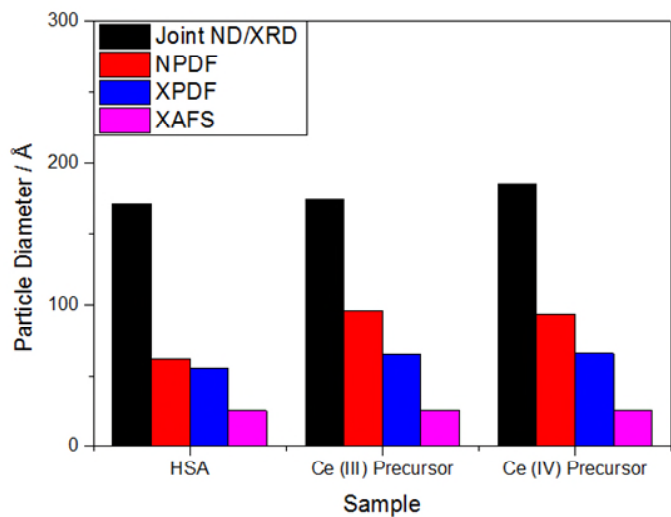

Figure 12: A comparison of particle sizes derived from different techniques.

The advantage of using the RMCProfile suite of software is that it takes simultaneously the Bragg diffraction data and the PDF data (one can define more than one r-range so that short and medium range order can be considered) and analyse them together to get the best model that fits both short, medium and long-range. For these samples, the $G(r)$ of the PDF data were separated into 2 distinct ranges, with one being fitted between 1 - $5 \AA$ and the other between $1-15 \AA$, in order to increase the weighting of the short range structure in the models.

The best fits obtained between experimental neutron diffraction data, NPDF, for two different ranges are shown in Error! Reference source not found. in the supporting Information. In all cases, the Bragg data and the short-range NPDF data are fitted very well. However, the quality of the fits decreases with increasing $r$. This is mainly due to the inability of the software to take into account the particle size effects and instrumental 
resolution when fitting in real space. Since the aim of this study is to probe local structure, this limitation is not severe.

The output of the RMCProfile analysis can be visualised by collapsing the $6 \times 6 \times 6$ supercells down onto the original unit cell, which overlays all the cerium and oxygen atoms together in "clouds" that are roughly equivalent to thermal ellipsoids generated from Rietveld refinement. The models generated based on this procedure are displayed in Figure 13.
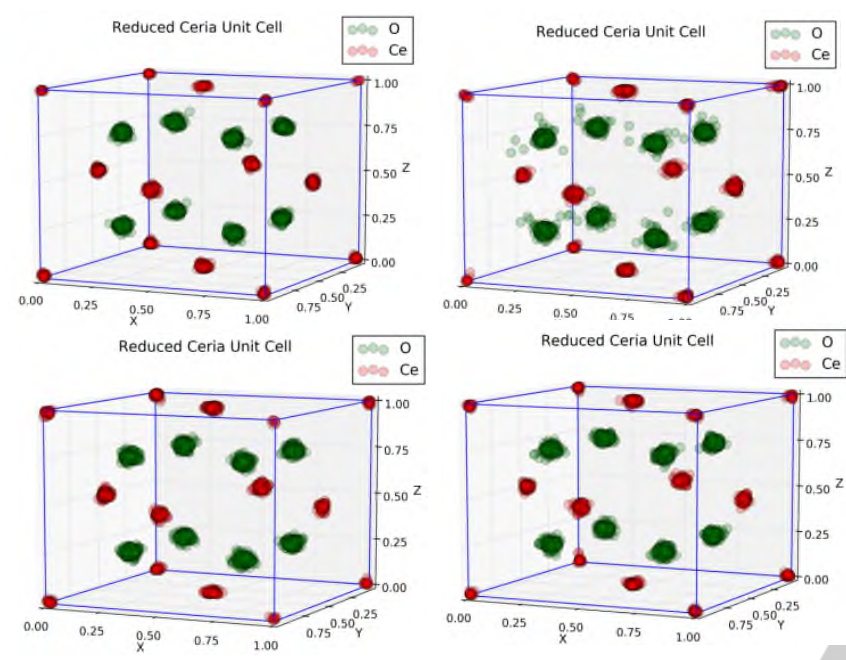

Figure 13: Reduced configuration to an overlaid single unit cell. Ceria NIST (Top left), High Surface Area Ceria (Top right) Ce (III) precursor (Bottom left), Ce (IV) precursor (Bottom right)

From Figure 13, we observe that the distribution of both Ce and $\mathrm{O}$ atoms are very narrow in the case of ceria NIST compared to the synthesised samples. In particular, the oxygen atoms in the HSA20 sample show maximum deviation from their ideal positions. In Error! Reference source not found. we summarise the average $\mathrm{Ce}-\mathrm{O}$ and $\mathrm{Ce}-\mathrm{Ce}$ distances related to first and second neighbour and mean square deviation of distances (MSD), determined from the RMC simulation taking into account every $\mathrm{Ce}$ atom in the box. MSD values of $\mathrm{Ce}-\mathrm{O}$ distances follow the trend HSA20> Ce (IV) precursor > Ce (III) precursor $>>$ NIST standard. Therefore, the disorder present in the oxygen could be one of the reasons for HSA20 to be more effective in oxygen mobility and catalytic processes.

Table 4: Calculated distances of atom pairs obtained from Rietveld analysis of diffraction data and RMCProfile analysis of total neutron total-scattering data. The relative mean square deviation from the final configurations produced in RMCProfile are given.

\begin{tabular}{lll|ll}
\hline \multirow{2}{*}{ Sample } & Atomic & Rietveld & \multicolumn{2}{|c}{ RMCProfile } \\
\cline { 2 - 5 } & Correlation & $\mathrm{R} / \AA$ & $\mathrm{R} / \AA$ & $\mathrm{MSD} / \AA^{2}$ \\
\hline \multirow{2}{*}{ Ceria } & Ce-O & 2.343 & 2.344 & 0.00675 \\
NIST & $\mathrm{Ce}-\mathrm{Ce}$ & 3.827 & 3.822 & 0.00659 \\
& $\mathrm{Ce}-\mathrm{O}$ & 2.343 & 2.343 & 0.00710 \\
$\mathrm{HSA}$ & $\mathrm{Ce}-\mathrm{Ce}$ & 3.825 & 3.821 & 0.00673 \\
$\mathrm{He} \quad$ (III) & $\mathrm{Ce}-\mathrm{O}$ & 2.344 & 2.344 & 0.00946 \\
Precursor & $\mathrm{Ce}-\mathrm{Ce}$ & 3.827 & 3.821 & 0.00934
\end{tabular}

\begin{tabular}{lll|ll} 
Ce (IV) & Ce-O & 2.344 & 2.344 & 0.0094 \\
Precursor & Ce-Ce & 3.827 & 3.821 & 0.00882 \\
\hline
\end{tabular}

\section{Summary}

In this work we have shown that the reliance on purely one form of characterisation technique may give only one viewpoint of the structure of the material, therefore utilising more than one technique is important to understand the material characteristics. All the techniques used show that no matter the synthesis procedure, the phase pure ceria fluorite structure is observed and the composition is close to stoichiometric $\mathrm{CeO}_{2}$. Combined Rietveld analysis of ND and XRD data shows that the HSA ceria, $\mathrm{Ce}$ (III) and $\mathrm{Ce}$ (IV) precursor ceria samples are more disordered than that of a ceria NIST standard. Neutron and Xray PDF studies support this observation. Dampening of the amplitudes of the PDFs of the synthesized samples is observed at $r>10 \AA$. This is indicative of a smaller crystallite size compared to the NIST sample that has an average crystallite

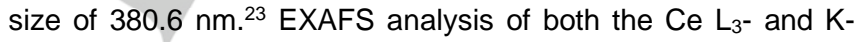
edges agrees with PDF analysis and Rietveld refinement of diffraction data showing crystallite sizes smaller than that of the NIST sample. EXAFS data analysis clearly indicates that the disorder is more in the oxygen site in all the synthesised samples compared to NIST standard and more significant disorder is seen for high-surface area material than other synthesised samples. It is difficult to say from these data whether the disorder associated with oxygen is related to the increased ratio of surface-oxygen in the smaller particles. More surface sensitive techniques that can determine structure near to the particle surface would be required to establish this. Analysis of the RMC models of all the materials further support the conclusion that more disorder is observed on the oxygen site for the ceria samples in comparison to the Ceria NIST material.

\section{Experimental}

\section{Sample Preparation}

Four ceria samples were investigated in this study. Ceria samples were prepared from the respective Ce (III) and Ce (IV) precursor salts. Cerium (III) nitrate hexahydrate solution $(60 \mathrm{ml})$, or diammonium cerium(IV) nitrate, $(60 \mathrm{ml})$, or were added to a stirred excess of aqueous ammonia (1L). The respective precipitates were stirred for $30 \mathrm{mins}$ and recovered by filtration. The precipitate was washed with water, dried at $105^{\circ} \mathrm{C}$ and calcined at $500^{\circ} \mathrm{C}$ for $2 \mathrm{hrs}$ in air. These calcined samples were referred in the text as "Ce (III) and Ce (IV) precursors". A commercially available Rhodia high surface area ceria (referred in the text as HSA) from Solvay was used after calcination to 
$500^{\circ} \mathrm{C}$ for 2 hours. A ceria Standard Reference Material ${ }^{\circledR}$ (SRM 674b) for XRD Quantitative Powder Diffraction from the National Institute of Standards \& Technology (NIST), Gaithersburg, MD was used 'as received'.

\section{X-ray and Neutron Total Scattering and Bragg diffraction}

All 4 samples were measured on POLARIS ${ }^{24}$ at the ISIS Facility, Rutherford Appleton Laboratory, UK. Data were collected using the low angle, $90^{\circ}$ and back scattering banks data over $28^{\circ}<2 \theta<42^{\circ}, 85^{\circ}<2 \theta<95^{\circ}$ and $130^{\circ}<2 \theta<160^{\circ}$ respectively. Data was collected in six 1 hour scans and later combined. The samples were ground into a fine powder and loaded into vanadium cans of $8 \mathrm{~mm}$ diameter. These cans were loaded onto an automatic sample changer and placed into the detector tank and the atmosphere was evacuated. The raw data were processed using MantidPlot. ${ }^{25}$

$X$-ray total scattering data of all the samples were collected on the 11-ID-B beamline at APS. The samples were ground into a fine powder and loaded into kapton tubes of $1 \mathrm{~mm}$ diameter. These kapton tubes were loaded into an automatic sample changer. Total scattering and Bragg diffraction patterns were collected using a Perkin Elmer Amorphous silicon 2D detector in air at room temperature. The diffraction patterns were collected using $\lambda=0.1430 \AA$; for Bragg diffraction data were collected between $2 \theta=0.0$ to $17.3^{\circ}$ and for total scattering patterns were collected over a Q-range $=0.7$ to $26 \AA^{-1}$. The raw data was processed using Fit2D. ${ }^{26}$

The analysis of the Bragg diffraction data (the Rietveld method $^{12}$ ) was performed using the graphical interface, EXPGUI ${ }^{27}$, of the GSAS software. ${ }^{28}$ The data correction for real space analysis was performed using the program GUDRUN 29 . The Fourier transformation of the $S(Q)$ data to produce the $G(r)$ was performed using the program STOG, distributed as part of the RMCProfile package. ${ }^{30}$ More details of the data collection and analysis are given in the supporting information. Hard sphere model was used for RMCProfile analysis.

\section{X-ray absorption spectroscopy (XAS) at $\mathrm{Ce}_{3} \mathrm{~L}_{3}$ and K-edges}

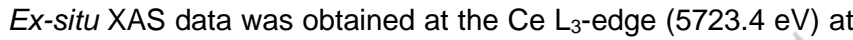
room temperature at $\mathrm{BM} 26 \mathrm{~A}^{31}$ beam line, at the European Synchrotron Research Facility (ESRF) which operates at $6 \mathrm{GeV}$ with a typical current of ca $100 \mathrm{~mA}$. Si (111) double crystal monochromator was used to collect the $\mathrm{L}_{3}$ edge data and all the data were collected in transmission mode using ionisation chambers. Approximately $10 \mathrm{mg}$ of sample was ground with approximately $90 \mathrm{mg}$ of fumed silica and made into a pellet and the XAS data were measured in step scans in an E-range of 5623.0 and $6154.0 \mathrm{eV}$. Ex-situ XAS data at the Ce K-edge $\left(40443 \mathrm{eV}\right.$ ) was obtained at room temperature on BM23, ${ }^{32}$ at the ESRF. The beamline uses a $\mathrm{Si}$ (311) double crystal monochromator and all the data were collected in transmission mode using ionisation chambers. Approximately $95 \mathrm{mg}$ of sample was ground in combination with approximately $15 \mathrm{mg}$ of cellulose and made into a pellet. The XAS data was measured in step scans in an E-range of 40200 and $41423 \mathrm{eV}$.

Analysis of the XAS data were performed using ATHENA and ARTEMIS software ${ }^{33}$. FEFF6L with Hedin-Lundqvist muffin-tin like potentials were used for all the scattering path calculations and further details of the analysis procedure are given in the supporting information.

\section{Acknowledgements}

We thank EPSRC and Johnson Matthey Plc for a Case Award (HRM and AHC). We thank the U.K. Science and Technology Facilities Council for allocating neutron beam time at the ISIS facility. We acknowledge the European Synchrotron Radiation Facility for provision of synchrotron radiation facilities. This research used resources of the Advanced Photon Source, a U.S. Department of Energy (DOE) Office of Science User Facility operated for the DOE Office of Science by Argonne National Laboratory under Contract No. DE-AC02-06CH11357. We also thank Peter Chupas for help with the beamline operations at APS.

\section{References}

(1) Farrauto, R. J.; Heck, R. M. Catal. Today 1999, 51, 351-360.

(2) Kašpar, J.; Fornasiero, P.; Hickey, N. Catal. Today 2003, 77, 419449.

(3) Catalysis by Ceria and Related Materials; Trovarelli, A., Ed.; Imperial College Press: London, 2002.

(4) Trovarelli, A.; Leitenburg, C. De; Boaro, M.; Dolcetti, G. Catal. Today 1999, 50, 353-367.

(5) Selvan, V. A. M.; Anand, R. B.; Udayakumar, M. ARPN J. Eng. Appl. Sci. 2009, 4, 1-6.

(6) Heck, R. M.; Farrauto, R. J. Appl. Catal., A 2001, 221, 443-457.

(7) Craciun, R.; Shereck, B.; Gorte, R. J. Catal. Lett. 1998, 51, 149-153.

(8) Pantu, P.; Kim, K.; Gavalas, G. R. Appl. Catal., A 2000, 193, 203214.

(9) Matatov-meytal, Y. I.; Sheintuch, M. Ind. Eng. Chem. Res. 1998, 37, 309-326.

(10) Walton, R. I. Prog. Cryst. Growth. Charact. Mater. 2011, 57, 93-108.

(11) Mamontov, E.; Egami, T. J. Phys. Chem. Solids 2000, 61, 13451356.

(12) Rietveld, H. M. J. Appl. Crystallogr. 1969, 2, 65-71.

(13) Takeshi, E.; Billinge, S. J. L. In Underneath the Bragg Peaks Structural Analysis of Complex Materials; Egami, T., Billinge, S. J. L., Eds.; Pergamon Materials Series; Pergamon, 2012; Vol. 16, pp 125.

(14) Yamazaki, S.; Matsui, T.; Ohashi, T.; Arita, Y. Solid State lonics 2000, 136-137, 913-920.

(15) Sears, V. F. Neutron News 1992, 3, 26-37.

(16) McMaster, W. H.; Del Grande, N. K.; Mallett, J. H.; H., H. J. Lawrence Livermore Lab., Rep. UCRL-50174 1969.

(17) Soldatov, A. V.; Ivanchenko, T. S.; Della Longa, S.; Kotani, A.; Iwamoto, Y.; Bianconi, A. Phys. Rev. B 1994, 50, 5074-5080.

(18) Paun, C.; Safonova, O. V; Szlachetko, J.; Abdala, P. M.; Nachtegaal, M.; Sa, J.; Kleymenov, E.; Cervellino, A.; Krumeich, F.; van Bokhoven, J. A. J. Phys. Chem. C 2012, 116, 7312-7317.

(19) Zhang, J.; Wu, Z.; Liu, T.; Hu, T.; Wu, Z.; Ju, X. J. Synchrotron Radiat. 2001, 8, 531-532.

(20) Chaboy, J.; Marcelli, A.; Tyson, T. A. Phys. Rev. B 1994, 49, 11652.

(21) Fonda, E.; Andreatta, D.; Colavita, P. E.; Vlaic, G. J. Synchrotron Radiat. 1999, 6 (1), 34-42.

(22) Price, S. W. T.; Zonias, N.; Skylaris, C.-K.; Hyde, T. I.; Ravel, B.; Russell, A. E. Phys. Rev. B 2012, 85, 75439. 
(23) No Title https://www-s.nist.gov/srmors/certificates/674B.pdf (accessed Aug 5, 2016).

(24) Hull, S.; Smith, R. I.; David, W. I. F.; Hannon, A. C.; Mayers, J.; Cywinski, R. Phys. B Condens. Matter 1992, 180-181, 1000-1002.

(25) Arnold, O.; Bilheux, J. C.; Borreguero, J. M.; Buts, A.; Campbell, S. I.; Chapon, L.; Doucet, M.; Draper, N.; Ferraz Leal, R.; Gigg, M. A.; Lynch, V. E.; Markvardsen, A.; Mikkelson, D. J.; Mikkelson, R. L.; Miller, R.; Palmen, K.; Parker, P.; Passos, G.; Perring, T. G.; Peterson, P. F.; Ren, S.; Reuter, M. A.; Savici, A. T.; Taylor, J. W.; Taylor, R. J.; Tolchenov, R.; Zhou, W.; Zikovsky, J. Nucl. Instruments Methods Phys. Res. Sect. A Accel. Spectrometers, Detect. Assoc. Equip. 2014, 764, 156-166.

(26) Hammersley, A. P.; Svensson, S. O.; Hanfland, M.; Fitch, A. N. Hausermann, D. High Press. Res. 1996, 14, 235-248.

(27) Toby, B. H. J. Appl. Crystallogr. 2001, 34, 210-213.

(28) Larson, A. C.; Von Dreel, R. B. Los Alamos Nation Lab. Rep. LAUR 1994, 86-748.

(29) Soper, $\quad$ A. $\quad$ K. No Title http://www.isis.stfc.ac.uk/instruments/sandals/dataanalysis/gudrun8864.html (accessed Oct 5, 2015).

(30) Tucker, M. G.; Keen, D. A.; Dove, M. T.; Goodwin, A. L.; Hui, Q. J. Phys. Condens. Matter 2007, 19, 335218.

(31) Nikitenko, S.; Beale, A. M.; Van Der Eerden, A. M. J.; Jacques, S. D. M.; Leynaud, O.; O’Brien, M. G.; Detollenaere, D.; Kaptein, R.; Weckhuysen, B. M.; Bras, W. J. Synchrotron Radiat. 2008, 15, 632640.

(32) Mathon, O.; Beteva, A.; Borrel, J.; Bugnazet, D.; Gatla, S.; Hino, R.; Kantor, I.; Mairs, T.; Munoz, M.; Pasternak, S.; Perrin, F.; Pascarelli, S. J. Synchrotron Radiat. 2015, 22, 1548-1554. Ravel, B.; Newville, M. J. Synchrotron Radiat. 2005, 12, 537-541. 
Entry for the Table of Contents (Please choose one layout)

Layout 1:

\section{ARTICLE}

Text for Table of Contents

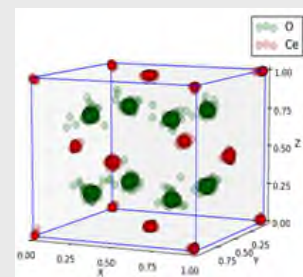

Layout 2:

\section{ARTICLE}

The structure of nano sized $\mathrm{CeO}_{2}$, was investigated using neutron and X-ray diffraction, total scattering and X-ray absorption spectroscopy at the $\mathrm{Ce}_{3}$ and K-edges. Detailed analysis of all this data indicate that the differences seen are primarily due to the particle size and disorder around oxygen sites; the particle size are estimated to be in the region of ca 5 $\mathrm{nm}$.
Huw R. Marchbank, ${ }^{a}$ Adam H. Clark, ${ }^{a}$ Timothy I. Hyde, ${ }^{b}$ Helen Y. Playford, ${ }^{c}$ Matthew G. Tucker, ${ }^{c, d}$ David Thompsett, ${ }^{b}$ Janet Fisher, ${ }^{b}$ Karena W. Chapman, ${ }^{e}$ Kevin A. Beyer, ${ }^{e}$ Manuel Monte, Alessandro Longo.g,h and Gopinathan Sankar ${ }^{\text {* }}$

Page No. - Page No.

Structure of nano-sized $\mathrm{CeO}_{2}$ material: a combined scattering and spectroscopic investigation
Huw R. Marchbank, Adam H. Clark, Timothy I. Hyde, ${ }^{b}$ Helen Y. Playford, ${ }^{c}$ Matthew G. Tucker, ${ }^{c, d}$ David Thompsett, Janet Fisher, ${ }^{b}$ Karena W. Chapman, ${ }^{e}$ Kevin A. Beyer, ${ }^{e}$ Manuel Monte ${ }^{f}$ Alessandro Longo. ${ }^{g, h}$ and Gopinathan Sankar ${ }^{\text {* }}$

Page No. - Page No.

Structure of nano-sized $\mathrm{CeO}_{2}$ material: a combined scattering and spectroscopic investigation 